\title{
SkeweR: a 3D Interaction Technique for 2-User Collaborative Manipulation of Objects in Virtual Environments
}

\author{
Thierry Duval, Anatole Lécuyer, Sébastien Thomas \\ IRISA, UMR 6074 \\ Campus de Beaulieu, 35042 Rennes Cedex \\ [Thierry.Duval, Anatole.Lecuyer]@irisa.fr
}

\begin{abstract}
This paper describes a novel 3D interaction technique called the "SkeweR", dedicated to the 2-user collaborative manipulation of objects in virtual environments. This technique enables two users to move simultaneously the same virtual object in 3D. For this aim, each user manipulates the object by one crushing point, like handling the extremity of a skewer. The SkeweR uses only translation information from the users' motions to change both the position and orientation of the manipulated object. By using more crushing points, this technique could easily be extended to 3 or more users. Thus, the SkeweR technique could be used to improve the collaborative manipulation of objects in numerous applications of Virtual Reality, such as: virtual prototyping, maintenance and training simulations, architectural mock-up reviews, etc.
\end{abstract}

CR Categories: H.5.2 [Information Interfaces and Presentation]: User Interfaces - Evaluation/methodology, Input devices and strategies, Interaction styles, User-centered design; H.5.1 [Information Interfaces and Presentation]: Multimedia Information Systems - Artificial, augmented, and virtual realities, Evaluation/methodology; H.1.2 [Information Systems]: User/Machine Systems - Human factors, Human information processing; I.3.6 [Computer Graphics]: Methodology and Techniques - Interaction techniques, Ergonomics.

Keywords: 3D interaction, Multiple Users, Collaborative Work, Manipulation, Skewer.

\section{INTRODUCTION}

Manipulation of objects is one of the most fundamental tasks of 3D interaction in Virtual Reality (VR) [3]. In addition, the collaborative manipulation of virtual objects by multiple users seems to be a very promising area for Collaborative Virtual Environments (CVE) [2]. The collaborative manipulation of objects seems indeed necessary in many different applications of VR such as for virtual prototyping, training simulations, assembly and maintenance simulations, etc [9].

In such collaborative tasks, all the users participate to the motion applied to the object manipulated in the VE. The separate motions of the different users are used to define the final motion of the virtual object. However, probably due to the complexity of the current VR interfaces, no universal solution has already been proposed for two distinct users to apply a motion to a unique manipulated object.
Therefore, in this paper we introduce a novel interaction technique for two users to move the same virtual object simultaneously in a VE. The technique is called the "SkeweR" technique since each user manipulates the object by one crushing point, like handling one extremity of a skewer. When using the SkeweR technique, the final motion of the manipulated virtual object is based on a combination of the two translation motions of the users.

The following paper begins with a description of related work in the field of collaborative manipulation of objects in virtual environments. Then it describes the concept of the SkeweR technique and its implementation. The paper ends with a conclusion and a description of potential perspectives.

\section{RELATED WORK}

The most frequent technique used in virtual environments for two users to interact with the same shared object remains unfortunately to allow only one user to manipulate the object at a time. However, recent studies upon highly collaborative manipulations have proposed novel 3D interaction techniques, which enable several users to interact with a shared object simultaneously [8][9].

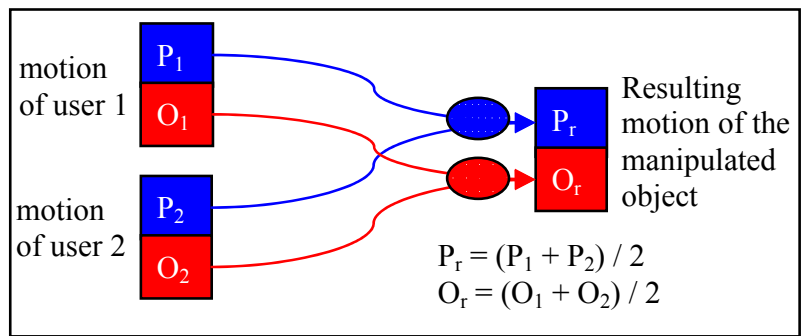

Figure 1: principle of the average technique of Ruddle et al. [9] (P stands for position, $O$ stands for orientation)

Ruddle et al. [9] have proposed several solutions to combine the movements of both users in order to obtain the final movement of the virtual object during a collaborative task in VR. First, they proposed to add the two motions (asymmetric integration of movements). Second, they proposed to compute the average of the two motions (see Figure 1), which basically results in dividing by two the amplitude of the addition motion aforementioned. Third, they proposed to keep only the common part (intersection) of the two motions (symmetric integration of movements). In this third case, the signed minimum value on each axis is kept. Thus, two identical motions along the same axis but with opposite directions will end into a null common part and a null motion.

The results of Ruddle et al. [8] showed that the average technique and the intersection technique were globally equivalent 
in terms of task completion time. However, the intersection technique was found better when the two users had to perform a similar action, for instance when moving a virtual piano in translation in a corridor. Conversely, the average technique was better when users had to perform different tasks, for instance turning the virtual piano in one corner of the corridor.

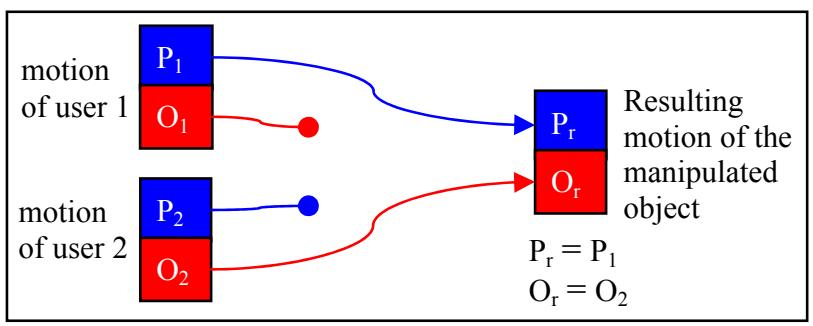

Figure 2: principle of a DOF separation technique (translations vs. rotations) [8]

Another way for two users to collaborate when moving a virtual object is to split the parts of the task among the users, as described by Pinho et al. [8]. In this case, the number of DOF (Dregrees Of Freedom) that each user can access and control is limited. The architecture of such an implementation (described in [8]) is displayed on Figure 2. In this case, one user controls the object's rotations while the other user controls the object's translations. Partial evaluations of this technique showed that the separation of DOF could be useful when users do not have the same view of the virtual scene, i.e. when they have two complementary views [8]. Thus, the separation of DOF seems useful when each user interacts with the DOF he/she can best observe.

A lot of innovative 3D interaction techniques have also been proposed in the field of manipulation of virtual objects with the two hands of a single user [5] [7]. Cutler et al. [5] gave a taxonomy of two-hand tools for 3D interaction. Among others, they described the "grab-an-carry" technique, which is a bimanual symmetric tool that allows to carry and turn an object around with both hands. Each hand can also be used independently as a one-hand grab tool which allows to pick-up a single object an move it freely. In their taxonomy, Cutler et al. [5] also described the "trackball" technique which can be considered as the equivalent (in the field of 2-hand 3D interaction techniques) of the 2-User DOF separation technique described above and displayed on Figure 2. The "trackball" technique is a bimanual asymmetric tool which allows to use the left hand to position a virtual object while the right hand rotates this object around its center.

In the field of haptic interaction, researchers have also developed some haptic techniques for 2 users to interact simultaneously with the same object using force-feedback devices. In such studies, each user is generally clutched to the manipulated object [1]. The force-feedback of the haptic devices is directly used to constrain the motions of the users and to apply commands on the manipulated object using simple physically based model. For instance, in the study of Basdogan et al. [1], the haptic interaction model simply simulates the translational movements of a ring on a wire and is not used to change the orientation of this ring.

\section{The SKewer TechniQue}

In this section, we describe a novel 3D interaction technique called the "SkeweR" technique, which allows multiple users to select and manipulate virtual objects simultaneously in a VE. Most of the previous interaction techniques were designed for the multiple users to manipulate the virtual object by translating or rotating its geometrical center (or gravity center). On the contrary, we propose to take into account the size and geometry of the object, in order to obtain a more natural interaction. Indeed, we propose to move the virtual object by grabbing any part of it, at any location.

The SkeweR technique can be first considered as an equivalent (in the field of 2-user collaborative manipulation) of the "graban-carry" tool described by Cutler et al. [5] which was designed for 2-hand manipulation. However, the principle of the SkeweR technique can also be extended, in order to be used by 3 (or more) users. The SkeweR technique can also be compared with the techniques developed for shared haptic interaction [1]. However, the SkeweR technique does not use force-feedback and physical models, but simply uses kinematics and information of motions of the users. The SkeweR technique can tolerate large offsets between the position of the user's hand (extremity of the device) and the position of the shared object in the simulation.

\subsection{Concept}

The SkeweR technique uses only the translation motion of the users. These motions are measured by position sensors. They are used to move one virtual 3D cursor per user in the VE. These 3D cursors are used to activate some "crushing points" at the surface of the manipulated object (see Figure 3). We will assume that a virtual $3 \mathrm{D}$ cursor stays at the same position than its associated crushing point.
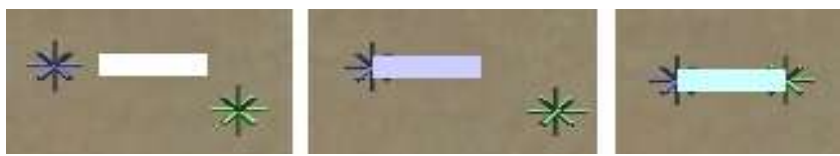

Figure 3: two users reach and crush a parallelepiped object with their virtual $3 D$ cursors

When associated with the crushing points, the 3D cursors of the two users become the extremities of a virtual skewer which is used to hold and move the interactive object. As displayed on Figure 4, a virtual object (parallelepiped) can be turned and rotated easily in a VE without using any rotation motion from the users.
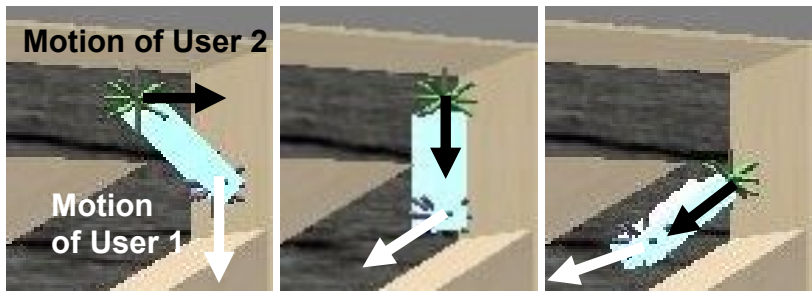

Figure 4: 2-user collaborative manipulation of an object with the SkeweR technique in one turn of a virtual maze

The SkeweR technique uses only the translation motions of the two users to move the objects within a complex 6 DOF 
movement. The scheme is thus different from the techniques described in section 2, making the SkeweR technique a hybrid technique, as displayed on Figure 5.

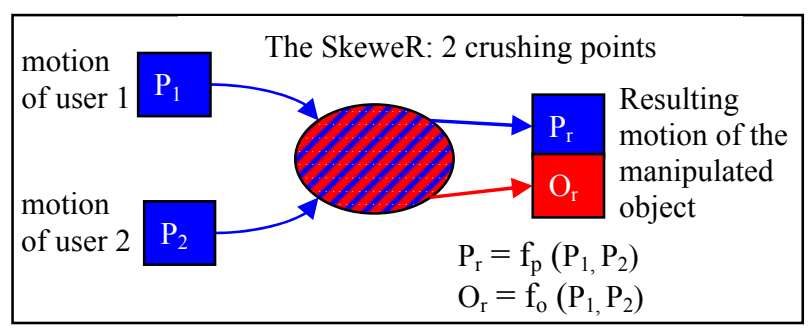

Figure 5: principle of the SkeweR technique

\subsection{Implementation of the SkeweR technique}

In this section, we will first present how it is possible to rotate and translate a virtual object thanks to only one crushing point. Then we will show how two users can use two crushing points. Last, we will describe how it is possible to extend the technique for 3 or more users.

\subsubsection{Changing the orientation of the manipulated object with one crushing point}

Once a crushing point (associated to a 3D cursor) is selected and activated at the surface of the manipulated object, the translation motions of the user are used to move the cursor and then to compute a new rotation increment for the object. At each time step, we consider an elementary straight motion of the cursor, and we compute an elementary rotation to apply to the object. Let us assume that Po is the geometrical center of the object, $\mathrm{Pc}_{\mathrm{t}}$ the position of the $3 \mathrm{D}$ cursor at time $\mathrm{t}, \mathrm{Pc}_{\mathrm{t}+1}$ the position of the $3 \mathrm{D}$ cursor at time $\mathrm{t}+1$. Then, the elementary rotation to apply to the interactive object will be a rotation of an angle $\theta$ around the $\omega$ axis, i.e. the axis which is passing by $\mathrm{Po}$ and orthogonal to the plane defined by $\mathrm{Po}, \mathrm{Pc}_{\mathrm{t}}$ and $\mathrm{Pc}_{\mathrm{t}+1}$. The angle $\theta$ and axis $\omega$ are computed as follows:

$$
\begin{gathered}
\theta=\operatorname{arcos}\left(\frac{\overrightarrow{P o P c_{t}} \cdot \overrightarrow{P o P c_{t+1}}}{\left\|\overrightarrow{P o P c_{t}}\right\| \times\left\|\overrightarrow{P o P c_{t}+1}\right\|}\right) \\
\vec{\omega}=\overrightarrow{P o P c_{t}} \wedge \overrightarrow{P o P c_{t}+1}
\end{gathered}
$$

\subsubsection{Changing the position of the manipulated object with one crushing point}

The change in position of the manipulated object is achieved by constraining the position of the crushing point (point located at the surface of the object) to follow the position of the cursor. Let us assume that $\mathrm{R}$ is the rotation used to determine the absolute orientation of the manipulated object. $\mathrm{R}$ is obtained by the accumulation of the elementary rotations defined previously with Equations 1 and 2. The new position of the object is then obtained using Equation 3. In this equation, $(\mathrm{Pc} / \mathrm{Po})$ represents the local position of the $3 \mathrm{D}$ cursor relatively to the center $(\mathrm{Po})$ of the manipulated object, in the relative frame of this object. This position is the same than the position of its associated crushing point, and it remains constant as long as the user does not choose to manipulate the object thanks to another crushing point.

$$
P o=P c-R \cdot\left(P c / P_{o}\right)
$$

As a result, this technique gives the user the impression that he/she is pulling the object with a virtual cord.

\subsubsection{Extension to 2 crushing points}

With two crushing points, each user may control the motion of the manipulated object as if he/she was holding one extremity of a virtual skewer. The final position of the interactive object is computed using Equation 4, in which Pc1 and Pc2 are the absolute positions of the cursors of the 2 users, and ( $\mathrm{Pc} 1 / \mathrm{Po})$ and $(\mathrm{Pc} 2 / \mathrm{Po})$ are the local positions of these $3 \mathrm{D}$ cursor relatively to the center of the manipulated object, in the relative frame of this object. The final position reached by the object corresponds to the middle of the two positions obtained when using the two crushing points alone.

$$
P o=\frac{1}{2}(P c 1+P c 2)-R \cdot \frac{1}{2}(P c 1 / P o+P c 2 / P o)
$$

Here again, the expression $((\mathrm{Pc} 1 / \mathrm{Po}+\mathrm{Pc} 1 / \mathrm{Po}) / 2)$ remains constant as long as one user does not choose to manipulate the object thanks to another crushing point.

Since the 4 points $P c 1_{t}, P c 1_{t+1}, P c 2_{t}$ and $P c 2_{t+1}$ do not always remain in the same plane, the final rotation applied to the interactive object now uses the vector $\mathrm{v}$ defined by Equation 5 . This vector is defined using the absolute position of the two cursors. The new rotation angle $\theta$ and the new rotation axis $\omega$ are then given respectively by Equation 6 and 7 .

$$
\begin{gathered}
\vec{v}=\overrightarrow{P c 1 P c 2} \\
\theta=\operatorname{arcos}\left(\frac{\overrightarrow{v_{t}} \cdot \overrightarrow{v_{t+1}}}{\left\|\overrightarrow{v_{t}}\right\| \times \| \overrightarrow{v_{t+1} \|}}\right) \\
\vec{\omega}=\overrightarrow{v_{t}} \wedge \overrightarrow{v_{t+1}}
\end{gathered}
$$

\subsubsection{With only 2 crushing points: one DOF is missing...}

It is not possible for the two users to rotate the virtual object around the axis of the skewer, since the rotation axis remains always orthogonal to the skewer's axis. To do so, the users have to stop their current motions and change the positions of their crushing points (=change the axis of the skewer).

However, we can imagine complementary techniques to make up for the lack of this sixth degree of freedom, and apply rotations around the skewer axis. First, we could use the rotation motions of the users around the skewer's axis straightforwardly, as measured by orientation trackers (similar to the "grab-andtwirl" tool referenced in [5]). However, this solution can not be implemented with 3 DOF trackers. Second, when pressing a button, users could be enabled to move their 3D trackers along the skewer's axis to define the sign and angle of a rotation as a function of the translation motion of each user along the axis. For example when the 3D trackers would get closer the object 
would rotate positively around this axis, and it would rotate negatively otherwise.

\subsubsection{Extension to 3 crushing points, or more...}

The proposed technique could be extended to the use of more crushing points. This could indeed allow a better control of the motion of the interactive object.

To control the orientation of an object, the best solution is to use 3 crushing points (not aligned), as these 3 points can easily be used to define exactly the orientation of the manipulated object. Indeed, these 3 crushing points define a plane embedded in the manipulated object and we can easily compute one vector orthogonal to this plane. Each time one crushing point (i.e. one $3 \mathrm{D}$ cursor) is moved, it is possible to compute a new orthogonal vector and to determine the exact rotation that transforms the initial vector into this new one. Furthermore, the position of this object could be computed as the average of the positions of the 3 crushing points. In the case of a manipulation of three crushing points by one user, one or two crushing points could be used to fix temporarily some position constraints, and the remaining crushing points would be used to move the object and change its orientation.

With four crushing points or more, the orientation of the interactive object might become over-constrained. Thus, with more than three crushing points, one must find arbitrary simplifications to apply changes in orientation to the object. Future work must be done to manage the computation of the orientation in this over-constrained case. However, the position of the interactive object still remains easy to compute, using the average position of all the crushing points.

\subsection{Preliminary experimental setup}

This technique has been implemented thanks to our OpenMASK platform $^{1}$ within our Reality Center, with magnetic trackers (see Figure 6). We have made some preliminary tests, asking two users to move a parallelepiped object inside a 3D maze.

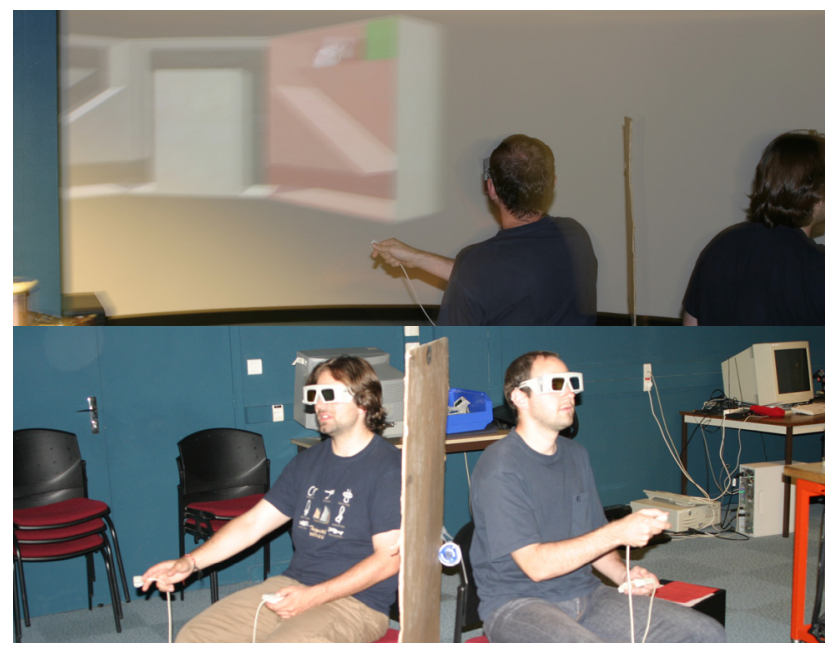

Figure 6: snapshots of the experimental setup. Each user holds 2 input devices: one $3 D$ tracker (dominant hand) and one "trigger" device with buttons (non-dominant hand)

\footnotetext{
${ }^{1}$ www.openmask.org
}

Our first observations indicate that the SkeweR technique seems very intuitive and natural to use. However, we must now perform a series of experiments to measure if this technique is easy and efficient enough for collaborative manipulations of $3 \mathrm{D}$ virtual objects.

\section{General Conclusion and Perspectives}

The SkeweR technique is a 3D interaction technique for 2-user collaborative manipulation of virtual objects. This technique enables two users to move simultaneously the same virtual object in a $3 \mathrm{D}$ environment. The technique uses and combines the translation motions of the two users, as measured by 3 DOF position trackers. Each user manipulates the object by one "crushing point", like handling the extremity of a skewer. This technique could also be used with only one crushing point, or also with 3 or more crushing points.

The SkeweR technique could be used to improve the manipulation of virtual objects in several applications of Collaborative Virtual Environments such as virtual prototyping, maintenance or training simulations.

Future work. Future work deals mainly with an evaluation of the SkeweR technique, as compared to the previous techniques described in the literature for the 2-user collaborative manipulation of virtual objects, i.e. the separation of DOF [8] and the average or intersection of the user's motions [9]. We would also like to study the use of three crushing points, with one, two or three users.

Acknowledgements. The authors would like to thank Dr. JeanMarie Burkhart for his valuable remarks.

\section{REFERENCES}

[1] Basdogan. C., Ho C.-H., Srinivasan M.A., Slater M., An Experimental Study on the Role of Touch in Shared Virtual Environments. ACM Transactions on Computer-Human Interaction, Vol. 7, Issue 4, Pages 443-460, December 2000.

[2] Benford S., Greenhalgh C., Rodden T., Pycock J., Collaborative Virtual Environments. Communications of the ACM, Pages 79-85, July 2001.

[3] Bowman. D., Kruijff E., LaViola J., and Poupyrev I., 3D User Interfaces: Theory and Practice. Addison-Wesley Eds, 2004.

[4] Bowman D., Interaction techniques for common tasks in immersive virtual environments. $\mathrm{PhD}$ thesis, Georgia Institute of Technology, June 1999.

[5] Cutler L.D., Frölich B., Hanrahan P., Two-Handed Direct Manipulation on the Responsive Workbench. ACM/SIGGRAPH Symposium on interactive 3D graphics, Providence, Rhode Island, United States, Pages 107-114, April 1997.

[6] Hand C., A Survey of 3D Interaction Techniques. Computer Graphics Forum, Pages 269-281, December 1997.

[7] Hinckley K., Pausch R., Proffit D., Kassel N.F., Two-Handed Virtual Manipulation. ACM Transactions on Computer-Human Interaction, Vol. 5, No. 3, Pages 260-302, September 1998.

[8] Pinho M., Bowman D., Freitas C., Cooperative Object Manipulation in Immersive Virtual Environments: Framework and Techniques. ACM Virtual Reality Software and Technology, HongKong, Pages 171-178, November 2002.

[9] Ruddle R., Savage J., Jones D., Symmetric and Asymetric Action Integration During Cooperative Object Manipulation in Virtual Environments. ACM Transactions on Computer-Human Interaction, Vol. 9, $\mathrm{N}^{\circ}$ 4, Pages 285-308, December 2002. 